\title{
Comparative genetic analysis of the novel coronavirus (2019-nCoV/SARS-CoV-2) receptor ACE2 in different populations
}

\author{
Yanan Cao $\mathbb{D}^{1}$, Lin Li', Zhimin Feng ${ }^{1}$, Shengqing Wan ${ }^{1}$, Peide Huang ${ }^{1}$, Xiaohui Sun ${ }^{1}$, Fang Wen ${ }^{1}$, Xuanlin Huang ${ }^{1}$, \\ Guang Ning ${ }^{1}$ and Weiqing Wang ${ }^{1}$
}

Dear Editor,

The ACE2 gene encodes the angiotensin-converting enzyme-2, which has been proved to be the receptor for both the SARS-coronavirus (SARS-CoV) and the human respiratory coronavirus NL63. Recent studies and analyses indicate that ACE2 could be the host receptor for the novel coronavirus 2019-nCoV/SARS-CoV-2 ${ }^{1,2}$. Previous studies demonstrated the positive correlation of ACE2 expression and the infection of SARS-CoV in vitro ${ }^{3,4}$. A number of ACE2 variants could reduce the association between ACE2 and S-protein in SARS-CoV or NL63. Therefore, the expression level and expression pattern of human ACE2 in different tissues might be critical for the susceptibility, symptoms, and outcome of 2019-nCoV/SARS-CoV-2 infection. A recent single-cell RNA-sequencing (RNA-seq) analysis indicated that Asian males may have higher expression of ACE2 $2^{6}$. Currently, the clinical reports of 2019$\mathrm{nCoV} / \mathrm{SARS}-\mathrm{CoV}-2$ infection from non-Asian populations for comparison are very limited. A study from Munich reported four German cases, all of which showed mild clinical symptoms without severe illness ${ }^{7}$. However, the genetic basis of ACE2 expression and function in different populations is still largely unknown. Therefore, genetic analysis of expression quantitative trait loci (eQTLs) ${ }^{8}$ and potential functional coding variants in ACE2 among populations are required for further epidemiological

\footnotetext{
Correspondence: Yanan Cao (caoyanan@vip.sina.com) or

Weiqing Wang (wqingw61@163.com)

${ }^{1}$ National Clinical Research Centre for Metabolic Diseases, State Key Laboratory of Medical Genomics, Shanghai Clinical Center for Endocrine and Metabolic Diseases, Shanghai Institute for Endocrine and Metabolic Diseases, Ruijin Hospital, Shanghai Jiaotong University School of Medicine, 200025 Shanghai, China
}

These authors contributed equally: Yanan Cao, Lin Li investigations of 2019-nCoV/SARS-CoV-2 spreading in East Asian (EAS) and other populations.

To systematically investigate the candidate functional coding variants in $A C E 2$ and the allele frequency (AF) differences between populations, we analyzed all the 1700 variants (Supplementary Table S1) in ACE2 gene region from the ChinaMAP (China Metabolic Analytics Project, under reviewing) and 1KGP (1000 Genomes Project) ${ }^{9}$ databases. The AFs of 62 variants located in the coding regions of $A C E 2$ in ChinaMAP, $1 \mathrm{KGP}$, and other largescale genome databases were summarized (Supplementary Table S2). All of the 32 variants potentially affecting the amino acid sequence of $A C E 2$ in databases were shown (Fig. 1a). Previous study showed that the residues near lysine 31 , and tyrosine $41,82-84$, and 353-357 in human ACE2 were important for the binding of S-protein in coronavirus ${ }^{5}$. The mutations in these residues were not found in different populations in our study. Only a singleton truncating variant of ACE2 (Gln300X) was identified in the ChinaMAP (Fig. 1a). These data suggested that there was a lack of natural resistant mutations for coronavirus S-protein binding in populations. The effects of low-frequency missense variants in populations for S-protein binding could be further investigated. The distributions of seven hotspot variants (Lys26Arg, Ile468Val, Ala627Val, Asn638Ser, Ser692Pro, Asn720Asp, and Leu731Ile/Leu731Phe) in different populations were shown (Fig. 1b). Six low-frequency loci (rs200180615, rs140473595, rs199951323, rs147311723, rs149039346, and rs73635825) were found to be specific in 1KGP database, the AFs of which were also low in the gnomAD and TopMed ${ }^{10}$ database. Only two of these six variants (rs200180615 and rs140473595) could be found in CHB (Han Chinese in Beijing) population with the $\mathrm{AF}<0.01$.

\section{(c) The Author(s) 2020}

(c) (i) Open Access This article is licensed under a Creative Commons Attribution 4.0 International License, which permits use, sharing, adaptation, distribution and reproduction cc) in any medium or format, as long as you give appropriate credit to the original author(s) and the source, provide a link to the Creative Commons license, and indicate if changes were made. The images or other third party material in this article are included in the article's Creative Commons license, unless indicated otherwise in a credit line to the material. If material is not included in the article's Creative Commons license and your intended use is not permitted by statutory regulation or exceeds the permitted use, you will need to obtain permission directly from the copyright holder. To view a copy of this license, visit http://creativecommons.org/licenses/by/4.0/. 


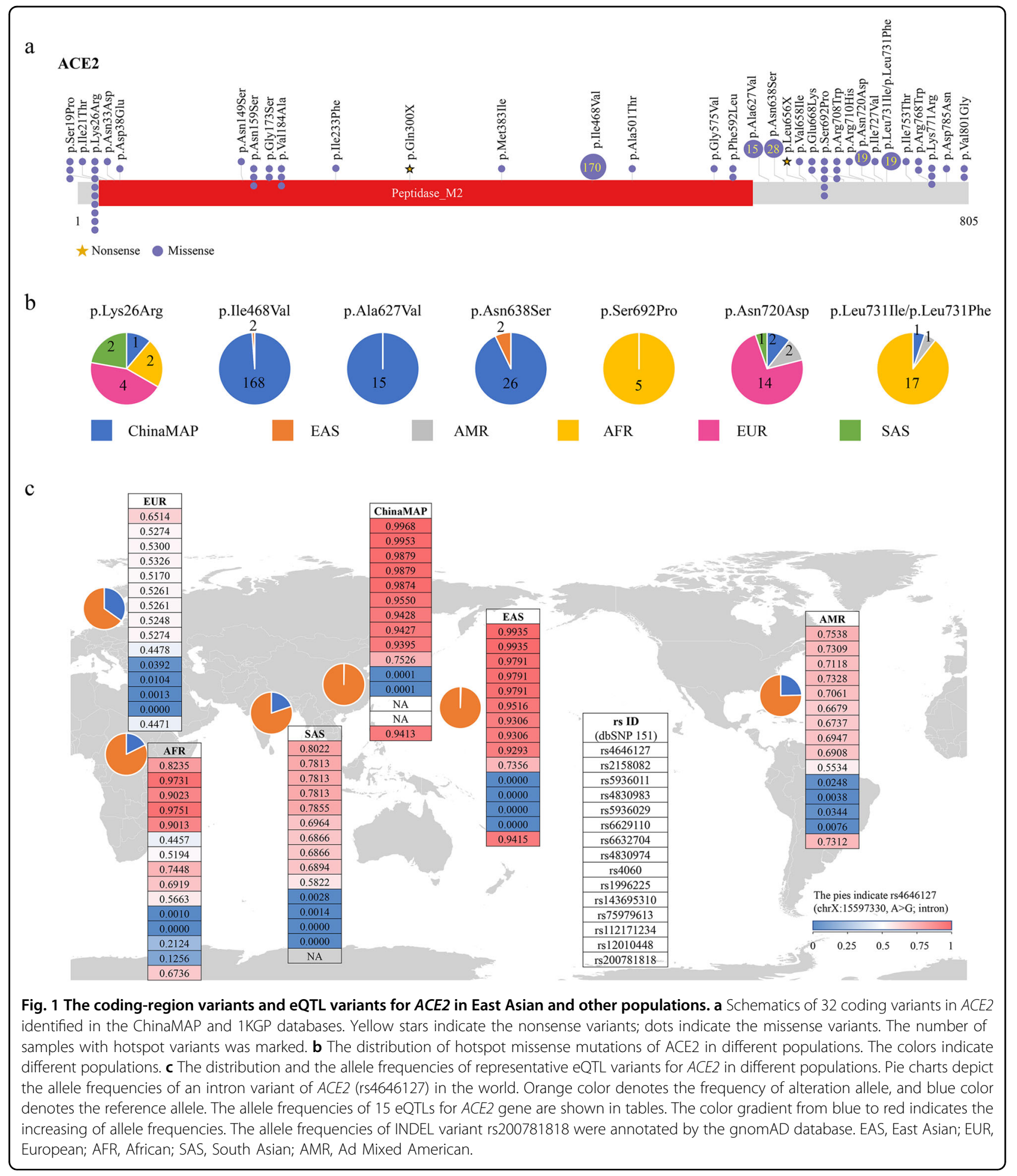

Interestingly, the SNP rs2285666 with the highest AF in the 62 variants exhibited much higher AF in the ChinaMAP (0.556) and CHS (Han Chinese South, 0.557) populations compared to others (AMR, Ad Mixed American, 0.336; AFR, African, 0.2114; EUR, European,
0.235). In addition, the homozygous mutation rate in males (0.550) was much higher than females $(0.310)$ in the Chinese population (Supplementary Table S2). Taken together, the differences in AFs of $A C E 2$ coding variants among different populations suggested that the diverse 
genetic basis might affect ACE2 functions among populations.

To analyze the distribution of eQTLs for $A C E 2$, we used the Genotype Tissue Expression (GTEx) database (https:// www.gtexportal.org/home/datasets). We found 15 unique eQTL variants (14 SNPs and 1 INDELs) for ACE2 with $q$ value $\leq 0.05$ in 20 tissues from the GTEx database (rs112171234, rs12010448, rs143695310, rs1996225, rs200781818, rs2158082, rs4060, rs4646127, rs4830974, rs4830983, rs5936011, rs5936029, rs6629110, rs6632704, and rs75979613). The AFs of the 15 eQTL variants were compared among different populations. Notably, our results showed most of the 15 eQTL variants had much higher AFs in the ChinaMAP dataset and EAS populations compared to European populations (Fig. 1c and Supplementary Table S3). The AFs of the top 6 common variants (rs4646127, rs2158082, rs5936011, rs6629110, rs4830983, and rs5936029) were higher than 95\% in EAS populations, whereas the AFs of these variants in European populations were much lower $(52 \%-65 \%)$. All of the 11 common variants $(\mathrm{AF}>0.05)$ and 1 rare variant (rs143695310) in the 15 eQTLs are associated with high expression of ACE2 in tissues (Supplementary Table S3). For instance, the eQTL variant rs4646127 (log allelic fold change $=0.314$ ), which locates in the intron of $A C E 2$ gene, has the highest AFs in both of the ChinaMAP (0.997) and EAS (0.994) populations. Comparatively, the AFs of rs4646127 in EUR (0.651) and AMR (0.754) populations are much lower. These findings suggested the genotypes of $A C E 2$ gene polymorphism may be associated higher expression levels of ACE2 in EAS population.

Recent reports of the ACE2 expression analysis in lung tissues from Asian and Caucasian populations are still controversial. The single-cell RNA-seq analysis reported that the Asian donor had much higher ACE2 expression cell ratio than white and African-American donors ${ }^{6}$. In contrast, the ACE2 expression analysis using the RNA-seq and microarray datasets from control lung tissues indicated there were no significant differences between Asian and Caucasian, or male and female ${ }^{11}$. The ACE2expressing cells are a very small part of cells in lung tissues $^{6}$. The sample size and the purity of ACE2-positive cells in the selected samples would influence the conclusions. Our analysis showed the differences in distribution and AFs of eQTLs for ACE2 in different populations, indicating the diversity of ACE2 expression pattern in populations (Supplementary Table S3). Largescale and multiple tissue-level analysis of single-cell RNAseq would be more accurate for the expression analysis of ACE2 in different populations. In addition, our data showed the moderate difference in AFs of eQTLs between South Asian and EAS, which suggests the potential difference of ACE2 expression in different populations and ethnics in Asia (Fig. 1c).
In summary, we systematically analyzed coding-region variants in $A C E 2$ and the eQTL variants, which may affect the expression of ACE2 using the GTEx database to compare the genomic characteristics of ACE2 among different populations. Our findings indicated that no direct evidence was identified genetically supporting the existence of coronavirus S-protein binding-resistant $A C E 2$ mutants in different populations (Fig. 1a). The data of variant distribution and AFs may contribute to the further investigations of ACE2, including its roles in acute lung injury and lung function ${ }^{12}$. The East Asian populations have much higher AFs in the eQTL variants associated with higher ACE2 expression in tissues (Fig. 1c), which may suggest different susceptibility or response to 2019-nCoV/SARS-CoV-2 from different populations under the similar conditions.

\section{Acknowledgements}

This work is supported by the National Basic Research Program of China (973 Program, 2015CB553601); the National Natural Science Foundation of China (81530020, 81522032).

\section{Author contributions}

Y.C. and W.W. conceived the study; L.L., Z.F, S.W., P.H., X.S., F.W., X.H., and Y.C. performed the bioinformatics analysis. G.N. contributed to the discussion of the results. Y.C. and L.L. wrote the manuscript; Y.C. and W.W. revised the manuscript.

Conflict of interest

The authors declare that they have no conflict of interest.

\section{Publisher's note}

Springer Nature remains neutral with regard to jurisdictional claims in published maps and institutional affiliations.

Supplementary Information accompanies the paper at (https://doi.org/ 10.1038/s41421-020-0147-1).

Received: 11 February 2020 Accepted: 16 February 2020

Published online: 24 February 2020

\section{References}

1. Zhou, P. et al. A pneumonia outbreak associated with a new coronavirus of probable bat origin. Nature https://doi.org/10.1038/s41586-020-2012-7 (2020).

2. Lu, R. et al. Genomic characterisation and epidemiology of 2019 novel coronavirus: implications for virus origins and receptor binding. Lancet https:/doi. org/10.1016/S0140-6736(20)30251-8 (2020).

3. Hofmann, H. et al. Susceptibility to SARS coronavirus $S$ protein-driven infection correlates with expression of angiotensin converting enzyme 2 and infection can be blocked by soluble receptor. Biochem. Biophys. Res. Commun. 319, 1216-1221 (2004).

4. Li, W. et al. The S proteins of human coronavirus NL63 and severe acute respiratory syndrome coronavirus bind overlapping regions of ACE2. Virology 367, 367-374 (2007).

5. Li, W. et al. Receptor and viral determinants of SARS-coronavirus adaptation to human ACE2. EMBO J. 24, 1634-1643 (2005).

6. Zhao, $Y$. et al. Single-cell RNA expression profiling of ACE2, the putative receptor of Wuhan 2019-nCoV. bioRxiv. https://doi.org/10.1101/2020.01.26. 919985 (2020).

7. Rothe, C. et al. Transmission of 2019-nCoV infection from an asymptomatic contact in Germany. N. Engl. J. Med. https://doi.org/10.1056/NEJMc2001468 (2020). 
8. The GTEx Consortium. The Genotype-Tissue Expression (GTEx) pilot analysis: multitissue gene regulation in humans. Science 348, 648-660 (2015).

9. Auton, A. et al. A global reference for human genetic variation. Nature 526, 68-74 (2015).

10. Taliun, D. et al. Sequencing of 53,831 diverse genomes from the NHLBI TOPMed Program. bioRxiv https://doi.org/10.1101/563866 (2019).
11. Cai, G. Tobacco-use disparity in gene expression of ACE2, the receptor of 2019-nCov. Preprint at https://doi.org/10.20944/preprints202002.0051.v1 (2020).

12. Imai, Y. et al. Angiotensin-converting enzyme 2 protects from severe acute lung failure. Nature 436, 112-116 (2005). 